\title{
Stellenwert der Positronen- Emissions-Tomographie mit 18-Fluor-Desoxyglukose (FDG-PET) in der Rezidivdiagnostik des Bronchialkarzinoms
}

\begin{abstract}
Zusammenfassung: Das Ziel der vorliegenden Untersuchung bestand darin, die diagnostische Aussagekraft der PositronenEmissions-Tomographie (PET) mit F-18-Desoxyglukose (FDG) im Rahmen der Rezidivdiagnostik von Patienten mit Bronchialkarzinomen zu bestimmen. Nach Injektion von $220 \pm 50 \mathrm{MBq}$ F-18Desoxyglukose wurde die Ganzkörper-PET (ECAT ART Scanner, Siemens (TI) durchgeführt. Die PET-Befundung durch visuelle Interpretation transversaler, koronaler und sagittaler Schnitte erfolgte im Konsens zweier erfahrener Nuklearmediziner ohne Kenntnis klinischer Daten oder anderer bildgebender Verfahren. 40 Patienten (= 41 Fälle) mit primär kurativ therapierten Bronchialkarzinomen wurden untersucht. In 29 der 35 Fälle mit Tumorrezidiv erfolgte eine pathologische Diagnosesicherung. Die FDG-PET-Untersuchung ergab einen richtig positiven Befund in 34/35 Fällen mit Tumorrezidiv. In 5/6 Fällen ohne Nachweis eines Tumorrezidivs ergab die PET-Untersuchung einen richtig negativen Befund. Die diagnostische Genauigkeit der FDG-PET lag insgesamt bei 39/41 = 95\% (95\%-Vertrauensintervall 83-99\%). Die FDG-PET weist eine hohe diagnostische Aussagekraft bei Rezidivverdacht von kurativ vorbehandelten Bronchialkarzinomen, kann jedoch die pathologische Diagnosesicherung nicht substituieren.
\end{abstract}

Evaluation of Positron Emission Tomography with 18Fluoro-2-Deoxyglucose (FDG-PET) in the Detection of Recurrent Lung Cancer: The aim of the present investigation was to evaluate the diagnostic accuracy of positron emission tomography with 18-fluoro-2-deoxyglucose (FDG-PET) in the detection of recurrent lung cancer. PET was performed using an ECAT ART scanner (Siemens CTI) after i. v. injection of $220 \pm 50 \mathrm{MBq}{ }^{18} \mathrm{FDG}$. PET data were analysed by visual interpretation of coronal, sagittal and transversal slices. PET scans were interpreted independently by two experienced nuclear medicine physicians without prior knowledge of the results of other imaging studies or clinical data. 40 patients (= 41 cases) who had undergone primarily curative tumour treatment, were evaluated. In 29 of 35 cases with recurrent tumour, diagnosis was verified by pathologic means. FDG-PET correctly identified tumour recurrence in 34/35 cases. In 5/6 cases without provent tumour recurrence PET gave true negative results. The overall accuracy of FDG-PET was 39/41 = 95\% (95\%-confidence interval $83-99 \%)$. FDG-PET shows high diagnostic accuracy in detecting

Pneumologie 2000; 54: 49-53

(c) Georg Thieme Verlag Stuttgart · New York ISSN 0934-8387
D. Ukena1', D. Hellwig'1, I. Palm, K. Rentz, M. Leutz, A. P. Hellwig ${ }^{1}$, C.-M. Kirsch ${ }^{1}$, G. W. Sybrecht

Medizinische Universitätsklinik, Innere Medizin V

${ }^{1}$ Abt. Nuklearmedizin, Universitätskliniken des Saarlandes, Homburg recurrent lung cancer in patients with prior curative tumour treatment, but cannot substitute the need for pathological diagnosis.

\section{Einleitung}

Im Unterschied zu den primär anatomisch-morphologisch orientierten Schnittbildverfahren wie Sonographie, Computertomographie und Magnetresonanztomographie bietet die Positronen-Emissions-Tomographie (PET) bei Verwendung geeigneter Tracer die Möglichkeit zur funktionellen Gewebscharakterisierung. So können mit der PET die regionale Gewebsdurchblutung, der Stoffwechsel, Rezeptorstrukturen oder die Gewebsretention von Pharmaka in Form von Schnittbildern dargestellt und regional quantitativ gemessen werden [17]. In onkologischer Sicht nutzt die PET den Tumorstoffwechsel und spezifische pathophysiologische Prinzipien zum Nachweis der Tumorlokalisation und der Tumorausdehnung aus.

Maligne Tumorzellen weisen im Vergleich zu gesunden Zellen eine deutlich gesteigerte aerobe und anaerobe Glykolyse auf. Es ist wahrscheinlich, dass der gesteigerte Glukosestoffwechsel ein sehr frühes Ereignis in der Entwicklung der malignen Transformation ist [9]. Die gesteigerte Glykolyse in Tumorzellen kann als ein in vivo messbarer Tumormarker angesehen werden. 2-Desoxyglucose (2-DOG) ist ein Glukose-Analogon, welches nach intrazellulärer Konversion zu 2-DOG-6-Phosphat in Tumorzellen nicht weiter metabolisiert werden kann [12]. 18-Fluor markiertes 2-Desoxyglukose (FDG) ist dasjenige Radiopharmakon, welches für die PET-Untersuchung zur in vivo-Erfassung des Glukosestoffwechsels von Tumorzellen geeignet ist (FDG-PET oder Onko-PET).

Der Stellenwert der FDG-PET in der klinischen Onkologie wird gegenwärtig in zahlreichen wissenschaftlichen Untersuchungen evaluiert. Beim Bronchialkarzinom sind als mögliche Indikationen für eine FDG-PET-Untersuchung der Nachweis des Primärtumors, die Bestimmung der Tumorausbreitung im Sinne eines Lymphknoten und Fernmetastasen-Stagings, das Ansprechen auf eine tumorspezifische Therapie und schließlich die Rezidivdiagnostik nach vorausgegangener Tumortherapie $z u$ nennen $[1,12,17,19]$. In der vorliegenden Arbeit werden unsere Erfahrungen mit FDG-PET in der Rezidivdiagnostik des Bronchialkarzinoms dargestellt. 


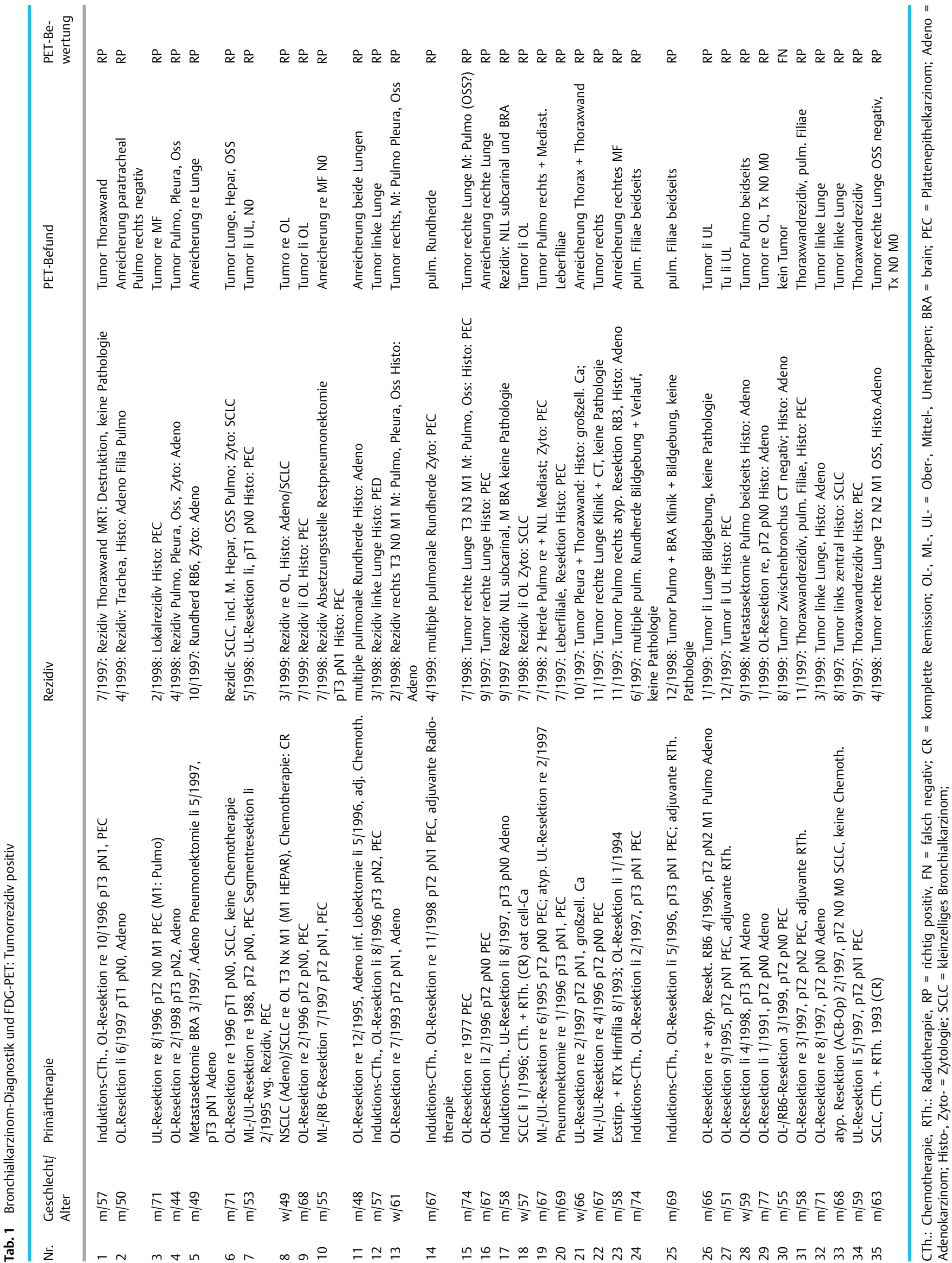




\section{Methodik}

Die vorliegende prospektive Untersuchung wurde im Zeitraum März 1997 - Juli 1999 bei 40 Patienten durchgeführt, die unter Verdacht auf ein Rezidiv eines Bronchialkarzinoms nach vorausgegangener kurativer Therapie (Operation, evtl. adjuvante zytostatische Chemotherapie oder Radiotherapie) oder nach mindestens über 15 Monate andauernder kompletter Remission nach zytostatischer Chemotherapie (evtl. kombiniert mit Radiotherapie) in stationärer Behandlung der Universitätskliniken des Saarlandes waren. Die Patienten gaben nach Aufklärung über Wesen, Bedeutung und Tragweite der Untersuchung ihr Einverständnis zur Durchführung der PET-Untersuchung.

Die PET-Untersuchung wurde in einem ECAT ART Scanner (Siemens-CTI) durchgeführt. Die Transmissionsmessungen erfolgten mit zwei externen ${ }^{137} \mathrm{Cs}$-Punktquellen (600 MBq). Den Patienten wurden in Nüchternzustand $220 \pm 50 \mathrm{MBq}$ F18-Desoxyglucose intravenös injiziert. Bei Patienten mit bekanntem Diabetes mellitus wurde entsprechend den Ausgangswerten die Blutzuckerkonzentration durch Injektion von Insulin unter den Schwellenwert von $160 \mathrm{mg} / \mathrm{dl}$ gesenkt. Die PET-Ganzkörperakquisition begann $90 \mathrm{~min}$ nach der Radionuklid-Injektion.

Die PET-Rekonstruktion erfolgte durch gefilterte Rückprojektion mit einem Hanning Filter (Zoomfaktor 1,5, Matrix $128 \times 128$, Grenzfrequenz 0,7 Nyquist). Die PET-Befundung durch visuelle Interpretation transversaler, koronarer und sagittaler Schnitte erfolgte im Konsens zweier erfahrener Nuklearmediziner ohne Kenntnis klinischer Daten oder anderer bildgebender Verfahren („einfach-blinde“ Auswertung). 95\%-Konfidenzintervalle für relative Häufigkeiten wurden gemäß der Binomialverteilung berechnet.

\section{Ergebnisse}

40 Patienten mit primär kurativ therapierten Bronchialkarzinomen wurden in der vorliegenden Untersuchung evaluiert. Unabhängig von der PET-Untersuchung wurde bei 35 Patienten ein Tumorrezidiv diagnostiziert (Tab.1). In den übrigen Fällen wurde kein positiver Nachweis eines Tumorrezidivs erbracht (Tab. 2). Ein Patient (aufgeführt als Nr. 17 der Tab. 1 und Nr. 38 der Tab. 2) wurde zweimal untersucht, entsprechend einmal mit einem negativen und einmal mit einem positiven Nachweis eines Tumorrezidivs.

Bei 7 Frauen und bei 28 Männern wurde ein Tumorrezidiv diagnostiziert (Tab.1). Histologisch handelte es sich in 4 Fällen um ein kleinzelliges Bronchialkarzinom (SCLC, small cell lung cancer), in einem Fall um ein gemischtzelliges Karzinom (Adenokarzinom und SCLC) und in 30 Fällen um ein nicht-kleinzelliges Bronchialkarzinom (NSCLC, non-small cell lung cancer), davon $n=17$ Plattenepithelkarzinome (PEC), $\mathrm{n}=11$ Adenokarzinome, $\mathrm{n}=1$ großzelliges Karzinom, $\mathrm{n}=1$ nicht genauer differenziertes NSCLC. In 24/35 Fällen bestand die Primärtherapie aus der chirurgischen Tumorresektion, welche immer als $\mathrm{R}_{0}$-Resektion gelang. 2 Patienten mit SCLC wurden ebenfalls primär operiert, erhielten jedoch keine adjuvante zytostatische Chemotherapie. In 6 Fällen wurde vor der chirurgischen Tumorresektion eine Induktionschemotherapie durchgeführt. Bei 2 Patienten erfolgte als primäre Intervention die extrapulmonale Metastasektomie, gefolgt von der Primärtumorresektion. Bei 3 Patienten wurde als Primärtherapie eine zytostatische Behandlung durchgeführt, davon wiederum 2 Fälle in Kombination mit einer perkutanen Radiotherapie. Auch in diesen Fällen wurde eine komplette Tumorremission (CR, complete remission) erzielt.

In 23/35 Fällen (66\%) wurde das vermutete Bronchialkarzinomrezidiv mittels histopathologischer Methoden gesichert. In weiteren 6 Fällen (17\%) gelang die Diagnosesicherung zytopathologisch. In den verbliebenen 6 Fällen erfolgte keine pathologische Diagnosesicherung des Tumorrezidivs. In diesen Fällen war entweder die Beurteilung anhand bildgebender Verfahren (z.B. multiple pulmonale Rundherde) eindeutig oder aber der Aufwand für die invasive pathologische Diagnosesicherung erschien wegen des Fehlens therapeutischer Konsequenzen als zu groß.

Die von anderen bildgebenden Verfahren unabhängige PETBefundung ergab einen Verdacht auf das Vorliegen eines Tumorrezidivs in 34 der 35 Fälle. Nur in einem Fall (Nr. 30) lieferte die PET ein falsch negatives Ergebnis. Hier handelt es sich um eine Tumorausbreitung im Zwischenbronchus, welche auch im Computertomogramm nicht diagnostiziert worden war. Die histologische Aufarbeitung der im Rahmen einer

Tab. 2 Bronchialkarzinom-Diagnostik und FDG-PET: Tumorrezidiv negativ

\begin{tabular}{|c|c|c|c|c|c|}
\hline $\mathrm{Nr}$. & $\begin{array}{l}\text { Ge- } \\
\text { schlecht/ } \\
\text { Alter }\end{array}$ & Primärtherapie & Rezidiv bzw. Diagnose & PET-Befund & $\begin{array}{l}\text { PET- } \\
\text { Bewer- } \\
\text { tung }\end{array}$ \\
\hline 36 & $\mathrm{~m} / 65$ & Pneumonektomie re 1984, pT2 pNO PEC & $\begin{array}{l}\text { 10/1998: im CT Tumor-Verdacht } \Rightarrow \text { Pneumonie, Infiltrat } \\
\text { rückläufig Zytologie negativ }\end{array}$ & kein Tumor & RN \\
\hline 37 & w/39 & OL-Resektion 12/1995 pT1 pN0 PEC & 7/1998: VD Rezidiv im CT Histologie: negativ & kein Rezidiv & RN \\
\hline 38 & $\mathrm{~m} / 57$ & $\begin{array}{l}\text { Induktions-CTh., UL-Resektion li 8/1997, } \\
\text { pT3 pN0 Adeno }\end{array}$ & $\begin{array}{l}\text { 10/1997: Ausschluss Rezidiv Klinik + Bildgebung, klin. } \\
\text { Verlauf }\end{array}$ & keine Anreicherung & RN \\
\hline 39 & $\mathrm{~m} / 71$ & UL-Resektion re 4/1992, pT2 pN0 Adeno & $\begin{array}{l}\text { 5/1997: Ausschluss Rezidiv Klinik + Bildgebung, Pathologie } \\
\text { negativ }\end{array}$ & keine Anreicherung & RN \\
\hline 40 & $\mathrm{~m} / 74$ & OL-Resektion li 3/1998, pT2 pN0 PEC & 2/1999: Ausschluss Rezidiv Bildgebung + Pathologie negativ & kein Tumor & RN \\
\hline 41 & $\mathrm{~m} / 57$ & OL-Resektion re 4/1998, pT2 pN1 M0 PEC & $\begin{array}{l}\text { 5/1998: Verdacht Rezidiv rechte Lunge Pathologie negativ, } \\
\text { bis 7/1999: kein Rezidiv }\end{array}$ & $\begin{array}{l}\text { Anreicherung rechte } \\
\text { Lunge }\end{array}$ & FP \\
\hline
\end{tabular}

RN = richtig negativ; FP = falsch positiv; für weitere Abkürzungen s. Tabelle 1 
starren Bronchoskopie entnommenen Schleimhautbiopsien aus dem Zwischenbronchus bestätigte die makroskopische Verdachtsdiagnose. Im Unterschied zu dem im Lungenresektat nachgewiesenen Plattenepithelkarzinom ergab die histologische Begutachtung des Tumors aus dem Zwischenbronchus den Nachweis eines Adenokarzinoms.

Bei weiteren 6 Patienten (1 Frau, 5 Männer) wurde nach primärer kurativer Tumorresektion anhand bildgebender und klinischer Verfahren ein Tumorrezidiv ausgeschlossen (Tab. 2). Die unabhängige PET-Befundung ergab ein richtig negatives Ergebnis in 5/6 Fällen. Nur in einem Fall lieferte die PET-Untersuchung ein falsch positives Ergebnis. Dieses beruhte am ehesten auf einer Fehlbeurteilung wegen einer entzündungsbedingten FDG-Akkumulation im Lungenparenchym. Auch im weiteren Verlauf ergab sich bei diesem Patienten kein Hinweis auf das Vorliegen eines Tumorrezidiv.

Insgesamt lag die diagnostische Genauigkeit der FDG-PET bezüglich der Rezidivdiagnostik bei 39/41 = 95\% (95\%-Konfidenzintervall $83-99 \%$ ).

\section{Diskussion}

Basierend auf den gegenwärtig verfügbaren Informationen aus kontrollierten Untersuchungen leistet die FDG-PET eine wertvolle Hilfestellung bei der Dignitätsbeurteilung des solitären Lungenrundherdes und von mediastinalen Lymphknoten $[8,10,18,22]$. Ein weiteres Indikationsgebiet für PET in der Onkologie ist die Rezidivdiagnostik nach vorheriger Primärtherapie. So wird beispielsweise auch außerhalb von klinischen Studien im Rahmen der Patientenversorgung der Einsatz von PET mit FDG als Radiopharmakon zur Rezidivdiagnostik von high grade Gliomen und mit einer geeigneten Aminosäure wie C-11 Methionin oder F-18 Tyrosin zur Rezidivdiagnostik von low grade Gliomen empfohlen $[3,6,14,15,17,21,23]$. Auch für die Rezidivdiagnostik des NSCLC wird ein klinischer Nutzen der FDG-PET postuliert $[17,19]$.

In der vorliegenden Arbeit wurden exklusiv nur kurativ vorbehandelte Patienten mit Bronchialkarzinomen ausgewertet. Bei $83 \%$ der Patienten wurde das Tumrorezidiv mit zytobzw. histologischen Methoden gesichert. In den übrigen Fällen bestand aufgrund der konventionellen bildgebenden Verfahren in Verbindung mit der klinischen Symptomatik kein

Tab. 3 Aussagekraft der FDG-PET bei der Rezidivdiagnostik des Bronchialkarzinoms: Literaturübersicht

\begin{tabular}{|c|c|c|c|c|}
\hline Autor & $\mathrm{n}$ & richtig positiv & richtig negativ & Genauigkeit \\
\hline $\begin{array}{l}\text { Duhaylongsod } \\
\text { et al. (1995) }\end{array}$ & 16 & $100 \%(6 / 6)$ & $100 \%(10 / 10)$ & $100 \%(16 / 16)$ \\
\hline $\begin{array}{l}\text { Hübner et al. } \\
\text { (1995) }\end{array}$ & 9 & $75 \%(3 / 4)$ & $80 \%(4 / 5)$ & $78 \%$ (7/9) \\
\hline $\begin{array}{l}\text { Inoue et al. } \\
\text { (1995) }\end{array}$ & 38 & $100 \%(>25 / 25)$ & $62 \%(8 / 13)$ & $87 \%(34 / 39)$ \\
\hline $\begin{array}{l}\text { Patz et al. } \\
\text { (1994) }\end{array}$ & 43 & $97 \%(34 / 35)$ & $100 \%(8 / 8)$ & $98 \%(42 / 43)$ \\
\hline eigene Daten & 41 & $97 \%(34 / 35)$ & $83 \%(5 / 6)$ & $95 \%(39 / 41)$ \\
\hline Gesamt & 148 & $97 \%(102 / 105)$ & $85 \%(35 / 42)$ & $93 \%(138 / 148)$ \\
\hline
\end{tabular}

Zweifel am Vorliegen eines Tumorrezidivs. Mit einer Ausnahme ergab die PET-Untersuchung eine gesteigerte Radionuklid-Anreicherung, welche mit dem Vorliegen eines pulmonalen Tumorrezidivs vereinbar ist. Der eine im PET als falschnegativ bewerteter Fall war auch im Thorax-CT nicht als Tumorrezidiv diagnostiziert worden.

Eine ähnlich hohe Treffsicherheit wie bei den richtig positiven Fällen zeigte die FDG-PET auch bei den Patienten, bei denen der initial geäußerte Verdacht auf das Vorliegen eines Rezidivs sich nicht bestätigte. Somit wies die FDG-PET in der vorliegenden Untersuchung eine hohe diagnostische Genauigkeit von 95\% (39/41 richtig positive Fälle) bei der Abklärung eines möglichen pulmonalen Tumorrezidivs eines Bronchialkarzinoms auf. Bei der Betrachtung dieser diagnostischen Genauigkeit muss jedoch die Patientenselektion berücksichtigt werden, da ausschließlich kurativ vorbehandelte Patienten untersucht wurden. Damit wurde ausgeschlossen, dass Therapiebedingte Gewebsänderungen oder Residualzustände nach vorausgegangener Therapie zu falsch-positiven FDG-Anreicherungen führen.

Die hohe diagnostische Genauigkeit der FDG-PET wurde auch in anderen Untersuchungen beschrieben. So wurde für die FDG-PET eine Genauigkeit von 92,5\% zur Detektion eines asymptomatischen Wiederauftretens von Bronchialkarzionomen bei 20 Patienten gefunden [5]. Hierbei wurden serielle PET-Untersuchungen über einen Zeitraum von 5 bis 40 Monaten nach Abschluss der Primärtherapie, welche überwiegend aus zytostatischer Chemotherapie und Radiotherapie bestand, durchgeführt. In einer anderen Untersuchung mit 38 Patienten wurden für die FDG-PET eine Sensitivität von $100 \%$ und eine Spezifität von 61,5\% für den Nachweis eines Tumorrezidivs bzw. eines Tumorresiduums errechnet [13]. Dabei erfolgte die PET-Befundung in Zusammenschau mit den Ergebnissen anderer bildgebender Verfahren.

In Tab. 3 sind in die der Literatur beschriebenen und von uns beobachteten Sensitivitäten und Spezifitäten der FDG-PETUntersuchung für die Rezidivdiagnostik des Bronchialkarzinoms zusammengefasst. Insgesamt wurden einschließlich der hier beschriebenen Ergebnisse 148 Fälle untersucht, an denen eine diagnostische Genauigkeit von 138/148=93\% (95\%Konfidenzintervall $89-97 \%$ ) gezeigt wurde $[4,11,13,16]$.

Die erwähnte Untersuchung von Inoue et al. [13] weist mit der relativ niedrigen Spezifität auf ein immanentes Problem der FDG-PET hin. Die Positronen-Emissions-Tomographie kann die pathologische Diagnosesicherung als Grundlage für Therapieentscheidungen nicht substituieren. Wie auch für die Rezidivdiagnostik gezeigt wurde, ist die semiquantitative PET-Auswertung durch Berechnung der SUV-Werte (standardized uptake value) zweifellos ein Fortschritt bei der Differenzierung von malignen und benignen Gewebsveränderungen $[5,7,13]$, jedoch naturgemäß nicht beweisend für das Vorliegen eines Tumorrezidivs. In ähnlicher Weise wurde auf die systemimmanente Limitation der FDG-PET auch bei der Differentialdiagnose maligner pleuraler Veränderungen hingewiesen $(2,20]$.

Zum gegenwärtigen Zeitpunkt liefert die FDG-PET im Kontext mit anderen bildgebenden Verfahren wichtige Zusatzinformationen in der Rezidivdiagnostik des Bronchialkarzinoms. In 
diesem Zusammenhang bekommt die FDG-PET möglicherweise einen besonderen Stellenwert in der Hilfestellung, den optimalen Ort zur Probenentnahme für die pathologische Diagnosesicherung festzulegen. Falls zu einem definierten Zeitpunkt eine pathologische Diagnosesicherung nicht möglich oder auch nicht notwendig ist, liefert möglicherweise auch die serielle PET-Untersuchung (Kontrolluntersuchungen in z.B. mehrmonatigen Abständen) hilfreiche Informationen für die Sicherung der Rezidivdiagnose. Diese Aspekte müssen jedoch in prospektiven, kontrollierten Untersuchungen bearbeitet werden.

\section{Literatur}

${ }^{1}$ American Thoracic Society/European Respiratory Society. Pretreatment evaluation of non-small-cell lung cancer. Am J Respir Crit Care Med 1997; 156: 320 - 332

2 Benard F, Sterman D, Smith RJ, Kaiser LR, Albelda SM, Alavi A. Metabolic imaging of malignant pleural mesothelioma with fluorodeoxyglucose positron emission tomography. Chest 1998; 114: $713-722$

${ }^{3}$ Di Chiro G, Oldfield E, Wright DC, De Michels D, Katz DA, Patronas NJ, Doppman JL, Larson SM, Ito M, Kufta C. Cerebral necrosis after radiotherapy and/or intraarterial chemotherapy for brain tumors: PET and neuropathologic studies. Am J Roentgenol 1988; 150: 189 - 197

${ }^{4}$ Duhaylongsod FG, Lowe VJ, Patz EF, Vaughn AL, Coleman RE, Wolfe WG. Detection of primary and recurrent lung cancer by means of F-18 fluorodexyglucose positron emission tomography. J Thorac Cardiovasc Surg 1995; 110: 130 - 139

${ }^{5}$ Frank A, Lefkowitz D, Jaeger S, Gobar L, Sunderland J, Gupta N, Scott W, Mailliard J, Lynch H, Bishop J, Thorpe P, Dewan N. Decision logic for retreatment of asymptomatic lung cancer recurrence based on positron emission tomography findings. Int J Radiation Oncology 1995; 32: 1495 - 1512

${ }^{6}$ Glantz MJ, Hoffman JM, Coleman RE, Friedman AH, Hanson MW, Burger PC, Herndon JE, Meisler WJ, Schold SC. Identification of early recurrence of primary central nervous system tumors by [18F] fluorodeoxyglucose positron emission tomography. Ann Neurol 1991; 29: 347 - 355

${ }^{7}$ Gupta N, Gill H, Graeber G, Bishop H, Hurst J, Stephens T. Dynamic positron emission tomography with F-18 fluorodeoxyglucose imaging in differentiation of benign from malignant lung/mediastinal lesions. Chest 1998; 114: 1105 - 1111

${ }^{8}$ Gupta C, Graeber GM, Rogers JS, Bishop HA. Comparative efficacy of positron emission tomography with FDG and computed tomographic scanning in preoperative staging of non-small cell lung cancer. Annals Surgery 1999; 229: 286 - 291

${ }^{9}$ Hiraki Y, Rosen OM, Birnbaum MJ. Growth factors rapidly induce expression of the glucose transporter gene. J Biol Chem 1988; 263: $13655-13662$

${ }^{10}$ Hoh CK, Schiepers C, Seltzer MA, Gambhir SS, Silverman DHS, Czernin J, Maddahi J, Phelps ME. PET in oncology: Will it replace the other modalities? Sem Nucl Med 19972794 - 106

${ }^{11}$ Hübner KF, Buonocore E, Singh SK, Gould HR, Cotten DW. Characterization of chest masses by FDG positron emission tomography. Clin Nucl Med 1995; 20: 293 - 298

${ }^{12}$ Hughes JMB. 18F-Fluorodexyglucose PET scans in lung cancer. Thorax 1996; 51: 16 - 22 (Suppl 2)

${ }^{13}$ Inoue T, Kim EE, Komaki R, Wong FCL, Bassa P, Wong W-H, Yang DJ, Endo K, Podoloff DA. Detecting recurrent or residual lung cancer with FDG-PET. J Nucl Med 1995; 36: 788 - 793

${ }^{14}$ Lilja A, Lundquist H, Olsson Y, Spannare B, Gullberg P, Langstrom B. Positron emission tomography and computed tomography in differential diagnosis between recurrent or residual glioma and treatment-induced brain lesions. Acta Radiol 1989; 30: 121 128

${ }^{15}$ Ogawa T, Kanno I, Shishido F, Inugami A, Higano S, Fujita H, Murakami M, Uemura K, Yasui N, Mineura K et al. Clinical value of PET with 18F-fluorodeoxyglucose and L-methyl-11C-methionine for diagnosis of recurrrent brain tumor and radiation injury. Acta Radiol 1991; 32: $197-202$

${ }^{16}$ Patz EF, Lowe VJ, Hoffmann JM, Paine SS, Harris LK, Goodman PC. Persistent or recurrent bronchogenic carcinoma with PET and 2[F-18]-2-deoxy-D-glucose. Radiology 1994; 191: 379 - 382

17 Reske SN, Bares R, Büll U, Guhlmann A, Moser E, Wannenmacher MF. Klinische Wertigkeit der Positronen-Emissions-Tomographie (PET) bei onkologischen Fragestellungen: Ergebnisse einer interdisziplinären Konsensuskonferenz. Nucl-Med 1996; 35: 42 - 52

18 Saunders CAB, Dussek JE, O’Doherty MJ, Maisey MN. Evaluation of fluorine-18-fluorodeoxyglucose whole body positron emission tomography imaging in the staging of lung cancer. Ann Thorac Surg 1999; 67: 790 - 797

${ }^{19}$ Schiepers C. Role of positron emission tomography in the staging of lung cancer. Lung Cancer 1997; 17: 29 - 35 (Suppl 1)

${ }^{20}$ Teirstein AS. Diagnosing malignant pleural mesothelioma. Chest 1998; 114: 666 - 667

${ }^{21}$ Valk PE, Budinger TF, Levin VA, Silver P, Gutin PH, Doyle WK. PET of malignant cerebral tumors after interstitial brachytherapy. Demonstration of metabolic activity and correlation with clinical outcome. J Neurosurg 1998; 69: 830 - 838

${ }^{22}$ Vansteenkiste JF, Stroobants SG, Lyen De PR, Bogaert J, Maes A, Deneffe GJ, Nackaerts KL, Verschakelen JA, Lerut TE, Mortelmans LA, Demedts MG. Lymph node staging in non-small cell lung cancer with FDG-PET scan: a prospective study on 690 lymph node stations from 68 patients. J Clin Oncol 1998; 16: 2142 2149

${ }^{23}$ Wienhard K, Herholz K, Coenen HH, Rudolf J, Kling P, Stocklin G, Heiss WD. Increased amino acid transport into brain tumors measured by PET of L-(2-18F)fluorotyrosine. J Nucl Med 1991; 32: $1338-1346$

Prof. Dr. med. D. Ukena

Medizinische Universitätsklinik

Innere Medizin $\mathrm{V}$

D-66421 Homburg

E-Mail: pnduke@med-rz.uni-sb.de 